Journal of Computer Science 5 (7): 511-518, 2009

ISSN 1549-3636

(C) 2009 Science Publications

\title{
Integrated Metric-Ad Hoc On-Demand Distance Vector: A Routing Protocol over Wireless Mesh Networks
}

\author{
Md. Arafatur Rahman, Farhat Anwar, Md. Saiful Azad \\ Department of Electrical and Computer Engineering, Faculty of Engineering, \\ International Islamic University Malaysia, Malaysia
}

\begin{abstract}
Problem statement: Wireless Mesh Networks (WMNs) is an emerging technology for wireless environment. Since WMNs are permanent or semi-permanent network, an efficient and reliable path establishment is the core concern for such type of networks. Several performance metrics has been designed for WMNs such as Expected Transmission Count (ETX), Expected Transmission Time (ETT), interference Aware Routing Metric (iAWARE), Link Type Aware (LTA) Metric, Success Probability Product (SPP) and so on. However, each of these individual routing metric considered some selected features thus a single metric is inadequate for selecting the most reliable path. Consequently, it is necessary to integrate multiple performance metrics into a routing protocol to attain optimal performance. Approach: In this study we proposed a technique of integrating multiple metrics to improve the performance of a WMN routing protocol. This technique was implemented in Ad hoc On-Demand Distance Vector (AODV) routing protocol thus leading to the development of Integrated Metrics AODV (IM-AODV) routing protocol. Results: The simulation results indicated that IMAODV protocol significantly outperformed the traditional AODV in WMNs environment. In a lightly loaded network the performance of IM-AODV is almost similar to AODV, however, in moderate to highly loaded network the performance of IM-AODV was improved by $10 \%$ (on average) compared to AODV. Conclusion/Recommendations: Incorporating multiple metrics in a routing protocol proved an effective mechanism for selecting the best path in a multi-hop wireless network (e.g. WMN) with loop free routing and avoiding highly loaded and lossy links. The proposed integrated metric scheme can also be considered for other routing protocols with simple modifications.
\end{abstract}

Key words: Wireless mesh networks, integrated metrics, AODV, IM-AODV, routing protocol

\section{INTRODUCTION}

These days, majority of the Internet accesses or connections are carried over wire line infrastructure employing DSL, T1 or cable-modem based connection. However, wire line infrastructures are more expensive and time consuming to set up than wireless infrastructures. Moreover, the Internet providers of the developing countries are not willing to install the necessary equipment such as optical fiber, copper-wire and other infrastructures for broadband services at the rural areas expecting marginal profit. The Wireless Network has emerged as a promising solution to overcome this crisis. It provides competitive data rate over wide areas for a large number of users compare to wired network. Moreover, it provides several facilities which include low cost equipment, ensure interoperability and reduce investment risk for operators.

The Wireless Mesh Networks (WMNs) are dynamically self-organization, self-configured and selfhealing, with the nodes in the network automatically establishing an ad hoc network and preserving the mesh connectivity ${ }^{[1]}$. Wireless mesh is functionally similar to the standard IEEE 802.11 infrastructure network with respect to its Basic Service Set (BSS) and Extended Service Set (ESS). The novelty is that, if the source and the destination station are not in the same BSS domain, the source Access Points (AP) does not forward the packet to all the APs in the ESS but the packet is sent along an AP's or station path to reach the destination station. The Wireless Distribution System (WDS) uses an extension of the IEEE 802.11 MAC/PHY to provide a protocol for auto configuring paths between Mesh

Corresponding Author: Farhat Anwar, Department of Electrical and Computer Engineering, Faculty of Engineering, International Islamic University Malaysia, Malaysia 
Points (MPs) in a multi-hop topology, supporting broadcast, multicast and unicast traffic.

Because of the features described above, WMNs are emerging rapidly. It supports several applications such as Broadband Home Networking, Community and Neighborhood Networking, Enterprise Networking, Metropolitan area networks, Disaster recovery and Security surveillance ${ }^{[2,3]}$. To date, several companies such as Intel $^{[4]}$, Microsoft ${ }^{[5]}$ and Motorola ${ }^{[6]}$, have already realized the potential of this technology and offer wireless mesh networking products. A few testbeds are already established in university research labs. However, there are still lots of issues need to be solved such as scalability, QoS assurance, better performance metrics, throughput improvement and considerable research efforts are still needed.

A number of wireless routing protocols are already designed to provide communication in wireless environment, such as AODV, OLSR, DSDV, ZRP, LAR, LANMAR, STAR, DYMO. However, routing protocol particularly suitable for WMNs has not designed been yet. Moreover, many enhancements over AODV have been proposed in the last couple of years, such as Stable Enhancement for AODV Routing Protocol $^{[7]}$, Ad-hoc On-Demand Multi-path Distance Vector Routing Protocol (AOMDV) ${ }^{[8]}$, Enhanced Metric Based Ad-hoc On Demand Distance Vector Protocol (EM-AODV) ${ }^{[9]}$, Multi-Link AODV (AODV$\mathrm{ML})^{[10]}$, Backup Routing with AODV (AODV-BR) ${ }^{[11]}$, Mobility Prediction Ad hoc On-Demand Multipath Distance Vector (MP-AOMDV) ${ }^{[12]}$ and many more. Nevertheless, integrated multiple metrics are not implemented on AODV to increase the performance yet. For this reason, in this research, multiple metrics are considered to get reliable path which will be appropriate for WMNs.

Some of the technical challenges in WMNs are optimal routing, load balancing, fairness, network auto configuration and mobility management. Main focus in this study is route optimization. Existing solutions in mobile ad hoc and sensor networks cannot be directly applied to WMNs due to the differences in traffic patterns, mobility scenarios, gateway functionalities and bandwidth requirements. Since most users in WMNs are primarily interested in accessing the Internet or other commercial servers, the traffic in WMNs is routed either toward the Internet Gateways (IGWs) or from the IGWs to clients. Thus, if multiple edges mesh routers choose the best throughput path toward a gateway, the traffic loads on certain paths and mesh routers increases tremendously thereby significantly decreasing the overall performance of the network. The routing algorithm therefore needs to determine routes between each traffic access point in a way that manage the entire mesh network while it is busy. Efficient routing mechanism can help in avoiding congestion and can increase the efficiency of the network resource utilization $^{[13]}$. Most of the traditional routing protocols designed for wired and wireless networks select path that minimize hop count ${ }^{[14-17]}$. However, hop count implicitly assumes that links either work well or don't work at all which is applicable for wired networks. This is not a reasonable approximation in the wireless case; since many wireless links have intermediate loss ratios, interference problem, low throughput. Douglas et al. ${ }^{[18]}$ explores the details of the performance of minimum hop count routing on a wireless test-bed and found that minimum hop count often finds route with significantly less throughput than the best available. They also proposed a high-throughput path metric for multi-hop wireless networks known as expected transmission count (ETX) metric. This is one of the first metrics that explicitly accounts for link quality during path selection. To compute ETX, each node broadcasts a probe packet every second. The probe contains the count of probes received from each neighboring node in the previous $10 \mathrm{sec}$. Based on these probes, a node can calculate the loss rate of probes on the links to and from its neighbors. Although, ETX performs very well in homogeneous single-radio environments, it does not perform as well in heterogeneous and multi-radio environments $^{[19]}$. Draves et al. ${ }^{[19]}$ improves ETX by considering the differences in link transmission rates. In Expected Transmission Time (ETT), with expected number of transmissions, packet size and raw bandwidth of the link is also considered. However, like ETX, ETT does not consider the presence of multiple channels and therefore, finds path with less channel diversity. To find paths with less intra-flow interference, Draves et al. ${ }^{[19]}$ proposed another performance metric known as Weighted Cumulative ETT (WCETT). One limitation of WCETT metric is that it explicitly consider inter-flow interference and when there are multiple flows in the network, it might finds a route in more congested areas of the network. The metric of interference and channel-switching $(\mathrm{MIC})^{[20]}$ is designed to consider the inter-flow (which is not considered in WCETT) and intra-flow interferences with load balancing capability. Although it provides better throughput and delay performance, it suffers from high overhead. Moreover, MIC does not guarantee is tonicity and therefore, when used with hop-by-hop routing protocols, it might forms routing loops ${ }^{[21]}$. Link Type Aware (LTA) is another routing metric which is proposed by ${ }^{[22]}$. It considers the infrastructure of WMNs and uses different link type 
among nodes to select suitable path. However, it does not consider the link quality and interference issues. Since each individual routing metric consider some features and it is difficult to satisfy all the requirements of WMNs by using a single metric, therefore, we propose an integrated metrics technique in this study. Four performance metrics are considered such as Expected Transmission Count (ETX), Round Trip Tome (RTT) and Life Time (LT) ${ }^{[23]}$ and traditional Hop Count, which grantees a minimum hop count with loop free routing and avoided highly loaded and lossy links.

\section{MATERIALS AND METHODS}

The basic functionality of the proposed protocol (IM-AODV) is very much similar to the AODV protocol. Only route discovery process makes the difference between these two protocols. Multiple metrics are integrated for selecting the reliable path in the proposed protocol. Similar to AODV, the enhanced protocol also uses the Route Request (RREQ), Route Reply (RREP) and Route Error packets for the route discovery and maintenance processes, except the Route Request and Reply packet formats are modified to carry additional information throughout the network.

Since the IM-AODV is the enhancement of AODV, the essential modifications performed are the addition of three fields to the Route Reply packet format, one additional field to the Route Request packet, as well as the modification of the routing table. The Route Error packet format is left unchanged. Similar to the AODV protocol, sequence numbers are used to ensure the freshness of the routes and avoiding routing loops in the network. The modification of routing table, route reply packet and route request packet are described in the following.

The routing table structure of this protocol is similar to AODV protocol; except three new entries named 'ETX', 'RTT' and 'LT'. The values in these fields contain the value of ETX, RTT and LT respectively. These values are needed to choose an optimum path.

When a node desires to communicate with a destination, it first checks its routing table for a route. Subsequently, the node always creates or updates a reverse route to the Source IP Address in its routing table. If a route to the Source IP Address already exists, it is updated only if either the Source Sequence Number in the RREQ is higher than the destination sequence number of the Source IP Addr in the route table, or the sequence numbers are equal, but the Integrated_Metrics_Value (IMV) in RREQ is smaller than the Prev_Integrated_Metrics_Value (PIMV) in the routing table. Integrated_Metrics_Value is calculated by using the following equation:
Integrated_Metrics_Value $=\left(\right.$ etx $/$ prev_etx $+\mathrm{rtt} / \mathrm{prev} \_r t t+$ hopCount / prev_hopCount - lifetime / prev_lifetime)

Prev_Integrated_Metrics_Value is calculated by using following equation:

Perv_Integrated_Metrics_Value $=$ (prev_etx $/$ etx + prev_rtt / rtt + prev_hopCount / hopCount - prev_lifetime $/$ lifetime)

Fig. 1 shows the format of the RREQ packet.

When a node wants to send a reply packet to the source, it first checks the routing table for collecting route information. The forward route for the destination is created or updated only if the Destination Sequence Number in the RREP is greater than the node's copy of the destination sequence number, or the sequence numbers are the same, but the route is no longer active or the Integrated_Metrics_Value in RREP is smaller than the Prev_Integrated_Metrics_Value in the route table entry. The Integrated_Metrics_Value and the Prev_Integrated_Metrics_Value are calculated similarly a the Route Request process. The format of the RREP is shown in Fig. 2.

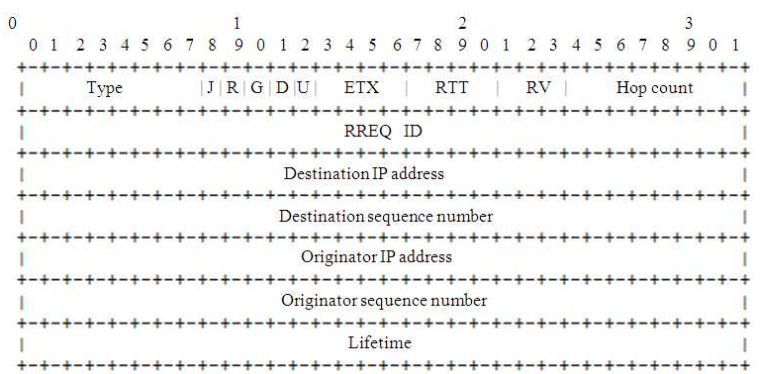

Fig. 1: Format of RREQ packet

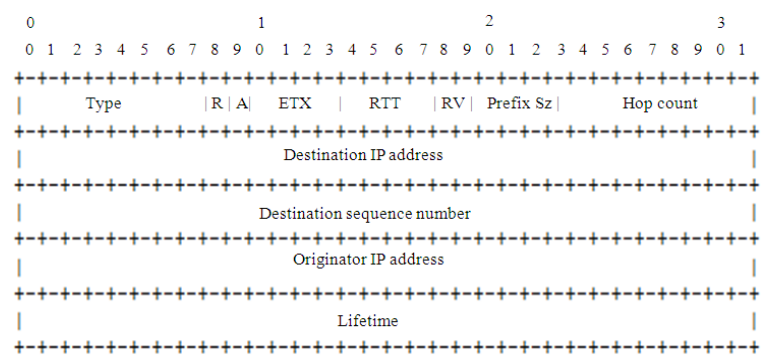

Fig. 2: Format of RREP packet 


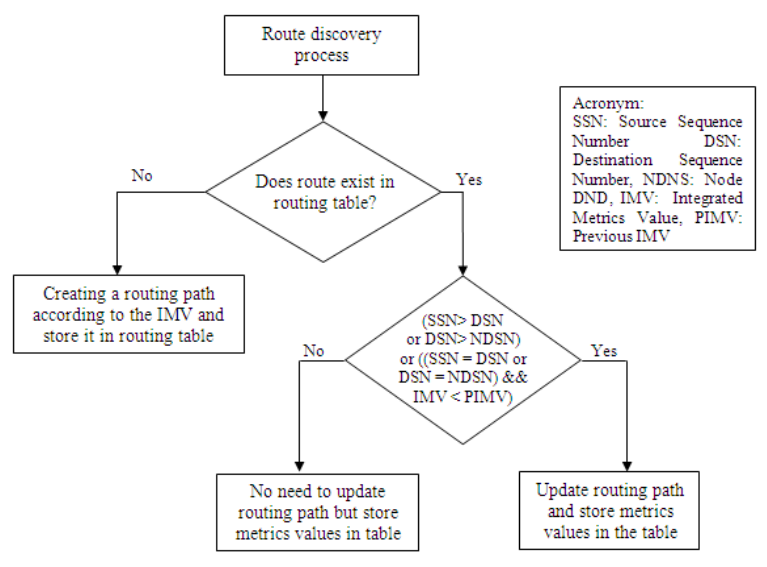

Fig. 3: Flow chart of route discovery process

The flow chart of route discovery process is demonstrated in Fig. 3.

When the source node wants to get best route to deliver data to the destination node, the following steps are followed:

- The source node first checks its routing table to find a route to the destination. If route (or routes) found, it selects route according to fresh routes or the optimal multiple metrics' values and sends the data to the destination

- If no route exists, it initiates a route discovery process. The source constructs a RREQ packet with the destination node. It then broadcasts the RREQ packet in the network

- Along the path each intermediate node on receiving the RREQ packet checks if it has an entry to the destination in its routing table. If the intermediate node has a route entry, it will send a route reply RREP packet back to the source along the current metrics values based on the following outcomes:

- If multiple entries to the same destination are found, it will select the route based on the multiple metrics

- If it doesn't have a route entry then it will rebroadcast the RREQ packet into the network

- After receiving one Route Request packet, the destination node will construct a Route Reply packet by appending the additional fields of RREP packet i.e., ETX and RTT values. The destination node will send multiple route replies until the timeout period has expired

- Each intermediate node along the forward path to the source will update the multiple metrics fields of RREP packet by comparing the multiple metrics value on the link on which the RREP was received and the values in the RREP packet header

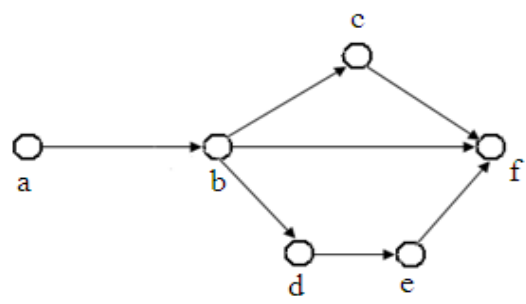

Fig. 4: A small Network architecture

Table 1: Integrated metrics value at low traffic

\begin{tabular}{llllll}
\hline & \multicolumn{3}{l}{ Routing metrics } & & \\
& ------- & & \\
Available route & Hop & ETX & RTT & LT & Value \\
\hline New path: [a-b-c-f] & 3 & 3 & 3 & 1 & IMV $=3.5$ \\
Old path: [a-b-f] & 2 & 2 & 2 & 1 & PIMV $=1.0$ \\
\hline
\end{tabular}

Table 2: Integrated metrics value at high traffic

\begin{tabular}{|c|c|c|c|c|c|}
\hline \multirow[b]{2}{*}{ Available route } & \multicolumn{5}{|c|}{ Routing metrics } \\
\hline & Hop & ETX & RTT & LT & Value \\
\hline New path: [a-b-c-f] & 3 & 3.00 & 3 & 1.0 & IMV $=0.74$ \\
\hline Old path: [a-b-f] & 2 & 4.66 & 5 & 0.5 & PIMV $=3.40$ \\
\hline
\end{tabular}

- The source node waits for a timeout period to receive multiple route replies. Storing all the routes in the buffer. Subsequently, it selects the best path based on the multiple metrics' values

For getting a clear idea of the proposed protocol, an example, shown in Fig. 4 can be considered.

In Fig. 4, "a" is a source and " $\mathrm{f}$ " is a destination node and, the network is in low traffic load. In the routing table the old route [a-b-f] is stored. However, if an alternative route [a-b-c-f] is available and the best route needs to be identified. Then, It is essential to calculate the value of IMV and PIMV. The IVM and PIMV are calculated by assuming metrics value, shown in Table 1, as follows:

$\mathrm{IMV}=3 / 2+3 / 2+3 / 2-1 / 1=3.5$

$\mathrm{PIMV}=2 / 3+2 / 3+2 / 3-1 / 1=1$

According to the proposed algorithm if PIMV is greater than IMV then the new route is selected. Since, it is false in this scenario then it will not update the route.

Now considering another scenario where the network load is high. Due to the network congestion the ETX and RTT value will be increased and life time will be decreased in the old route. For replacing the old route the IMV and PIMV value need to be recalculated. Assuming the metrics value, which are shown in Table 2, the updated value of IMV and PIMV will be the following: 
$\mathrm{IMV}=3 / 2+3 / 4.66+3 / 5-1 / 0.5=0.74$

$\mathrm{PIMV}=2 / 3+4.66 / 3+5 / 3-0.5 / 1=3.4$

Based on the proposed algorithm since PIMV is greater than IMV then the new route is selected. However, the traditional AODV will continue to use the old route. Thus the overall performance of the network will be significantly improved.

The existing AODV uses only one metrics for selecting the route which is Hop Count. It also uses Life Time to determine the expiration or deletion time of the route. However, we consider this lifetime as a metric which ensures a path with maximum life time. Along with Hop count and Lifetime we have chosen other two metrics such as ETT and RTT which are assisting to captures the effects of both packet loss ratios and path length and to avoid highly loaded or lossy links respectively. In addition, ETX is also an isotonic routing metric, which guarantees easy calculation of minimum weight paths and loop-free routing. The ETX metric's value in the node is calculated by using the following equation:

ETX $=$ Previous ETX $+(1 /(1-((10-$ count $) / 10)))$

Where:

Previous ETX = The stored value in the table

count $=$ The number of probes received from each neighboring node in the previous $10 \mathrm{sec}$

Subsequently, the RTT value is calculated by using the following equations:

RTT $=$ Previous RTT + GetSimTime $($ node $)$

Where:

Previous RTT = The stored value in the table

GetSimTime $($ node $)=$ The function which calculated the delay time of current node

Here we do not consider the traditional RTT. We only use a function to get the delay time of each node. Therefore, we can avoid the extra overhead which is generated by sending packet from source to destination to calculate traditional RTT. By using this four routing metrics we can get a reliable path which grantees a minimum hop count with loop free routing that avoids highly loaded and lossy links.

\section{RESULTS AND DISCUSSION}

The simulations have been performed using QualNet version 4.5, a software that provides scalable simulations of Wireless Networks and a commercial version of GloMoSim. In our simulation, we consider a network of 100 nodes that are placed randomly within a $1000 \times 1000 \mathrm{~m}$ area and operating over $500 \mathrm{sec}$. Multiple runs with different seed numbers are conducted for each scenario and collected data is averaged over those runs.

A two-ray propagation path loss model is used in our experiments with lognormal shadowing model. The transmission power of the routers is set constant at $20 \mathrm{dBm}$ and the transmission range of the routers is $250 \mathrm{~m}$. The data transmission rate is $2 \mathrm{Mbits}^{-1}$. At the physical layer 802.11 b and at MAC layer 802.11.s protocols are used. The traffic source is implemented using Constant Bit Rate (CBR). The packet size without header is 512 bytes. The length of the queue at every node is 50 Kbytes where all the packets are scheduled on a First-In-First-Out (FIFO) basis.

To evaluate the performance of routing protocols, both qualitative and quantitative metrics are needed. Most of the routing protocols ensure the qualitative metrics. Therefore, we use four different quantitative metrics to compare the performance. They are:

- $\quad$ Packet delivery ratio: The fraction of packets sent by the application that are received by the receivers ${ }^{[24]}$

- Jitter: Jitter is the variation in the time between packets arriving, caused by network congestion, timing drift, or route changes

- Average end-to-end delay: End-to-end delay indicates how long it took for a packet to travel from the source to the application layer of the destination $^{[25]}$

- Throughput: The throughput is defined as the total amount of data a receiver $R$ receives from the sender divided by the times it takes for $\mathrm{R}$ to get the last packet ${ }^{[26]}$

The performance differentials in this simulation are investigated using varying traffic load for 30 receivers. Traffic load is varied from 10-80 packets $\mathrm{sec}^{-1}$ and incremented by 10 packets $\mathrm{sec}^{-1}$. The results gained from simulations are illustrated in Fig. 5-8.

In Fig. 5, packet delivery ratio obtained for AODV and IM-AODV (Integrated Metric on AODV) is shown. At the beginning when traffic load was less e.g., 10 packets $\mathrm{sec}^{-1}$, both protocols display high packet delivery ratio and it declines with the increasing traffic load. Since integrated metrics mechanisms assist to avoid congestion and increase network resource utilization, IM-AODV performed significantly better than AODV when traffic load is higher. 


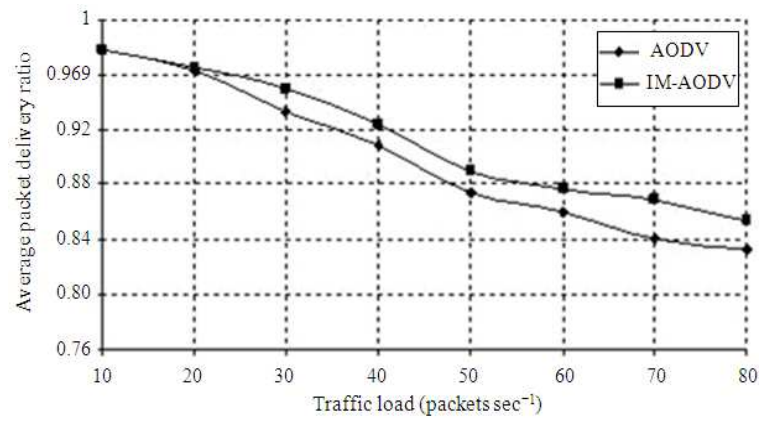

Fig. 5: Packet delivery ratio, for 30 receivers

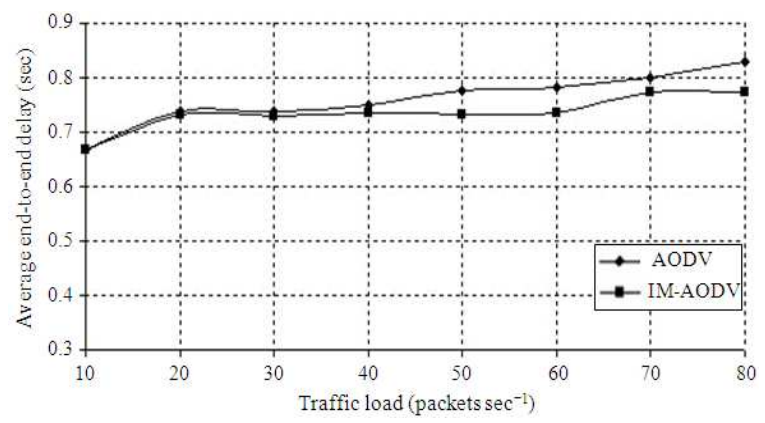

Fig. 6: End-to-end delay, for 30 receivers

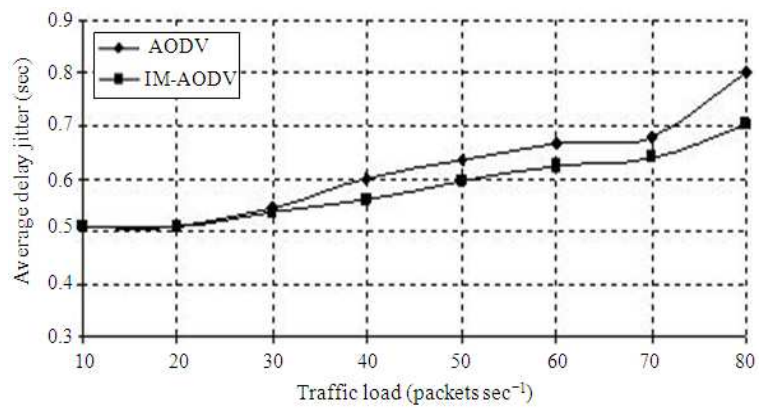

Fig. 7: Delay Jitter, for 30 receivers

Figure 6 demonstrates the average end-to-end delay of packets to travel from source to destination's application layer. It can be observed that end to end delay of IM-AODV is better than AODV. At low traffic load, both perform identical. However, with increasing traffic load, performance of IM-AODV elevate than AODV, since packets are transmitted over stable path in IM-AODV.

Figure 7 shows the delay jitters for AODV and IMAODV. Alike end to end delay, AODV has higher delay jitter than the IM_AODV, because of its higher congestion. The more latency suggests worse congestion.

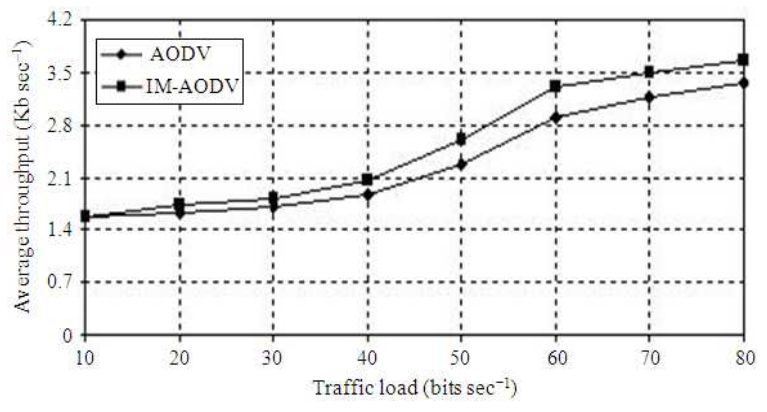

Fig. 8: Throughput, for 30 receivers

Figure 8 shows the throughput comparison of AODV and IM-AODV. The general observation from the simulation is that for throughput, IM_AODV and AODV perform similar in less "stressful" circumstances (lower traffic load). Conversely, IMAODV outperforms AODV in more stressful circumstances. The poor throughput performance of AODV is caused by its stale route problem.

For all the cases, it can be observed that in a lightly loaded network the performance of IM-AODV is almost similar to AODV, however, in moderate to highly loaded network the performance of IM-AODV is improved by $10 \%$ (on average) compared to AODV.

\section{CONCLUSION}

In this study, an improved performance metric based protocol named by IM-AODV has proposed for WMNs which will able to select more reliable path. Proposed performance metric is implemented in IM-AODV. Since WMNs is a semi-permanent network, the reliable path is a key issue here. The main goal of IM-AODV is to ensure a reliable path. Simulation result shows that IMAODV performs better than traditional AODV.

\section{ACKNOWLEDGMENT}

The researchers would like to acknowledge the Research Management Centre of IIUM for supporting this research work.

\section{REFERENCES}

1. Akyildiz, I.K. and X. Wang, 2005. A survey on wireless mesh networks. IEEE. Radio Commun., 43: S23-S30. DOI: 10.1109/MCOM.2005.1509968

2. Nguyen, U.T., 2008. On multicast routing in wireless mesh networks. Comput. Commun., 31: 1385-1399.

http://portal.acm.org/citation.cfm?id=1365162 
3. Akyildiz, I.K., X. Wang and W. Wang, 2005. Wireless mesh networks: A survey. Comput. Networks, 47: 445-487. DOI: 10.1016/j.comnet.2004.12.001

4. Cherry, S., 2007. Wi-Fi nodes to talk amongst themselves. IEEE Spectrum. http://tfa.rice.edu/news_n_media/spectrum.pdf.

5. Gkantsidis, C., B. Radunovic, P. Key, S. Gheorgiu, W. Hu and P. Rodriguez, 2007. Multipath Code Casting for Wireless Mesh Networks. Microsoft Technical Report MSR-TR-2007-67. ftp://ftp.research.microsoft.com/pub/tr/TR-2007-68.pdf

6. White, W., 2006. Mesh Networks: A Revolution in Wireless Connectivity. Motorola Technology Position Paper.

http://www.zdnet.com.au/whitepaper/0,200006332 8,22129626p-16001437q,00.htm

7. Zhong, X., S. H. Mei, Y. O. Wang and J. Wang, 2003. Stable enhancement for AODV routing; protocol. Proceeding of the 14th International Symposium on Persona1, lndoor and Mobile Radio Communication, Sept. 7-10, IEEE Xplore Press, USA., pp: 201-205. DOI: 10.1109/PIMRC.2003.1264261

8. Marina, M.K. and S.R. Das, 2001. On-demand multi path distance vector routing in ad hoc networks. Proceeding of the 9th International Conference on Network Protocols, Nov. 11-14, IEEE Computer Society, Washington, DC., USA., pp: 14-23.

http://portal.acm.org/citation.cfm?id=881590

9. Thanthry, N., S.R. Kaki and R. Pendse, 2006. EMAODV: Metric based enhancement to AODV routing protocol. Proceeding of the Vehicular Technology Conference, Sept. 25-28, IEEE Xplore Press, Montreal, Que., pp: 1-5. DOI: 10.1109/VTCF.2006.534

10. Pirzada, A.A. and R. Wishart, 2007. Multi-linked AODV routing protocol for wireless mesh networks. Proceeding of the IEEE Globe Telicommunications Conference, Nov. 26-30, IEEE Xplore Press, Washington, DC., pp: 4925-4930. DOI: 10.1109/GLOCOM.2007.934

11. Lee, S.J. and M. Gerla, 2000. AODV-BR: Backup routing in ad hoc networks. Proceedings of the IEEE Conference on Wireless Communications and Networking, Sept. 23-28, IEEE Xplore Press, Chicago, IL., USA., pp: 1311-1316. DOI: 10.1109/WCNC.2000.904822

12. Sambasivam, P., A. Murthy and E. Belding-Royer, 2004. Dynamically adaptive multipath routing based on AODV. Proceeding of the MedHocNet, June 2004, Bodrum, Turkey, pp: 106-117. http://www.ece.osu.edu/medhoc04/medhocnetfiles/ papers/S03.2.pdf
13. Liang, M. and M.K. Denko, 2007. A routing metric for load-balancing in wireless mesh networks. Proceeding of the 21st International Conference on Advanced Information Networking and Applications Workshops, May 21-23, IEEE Xplore Press, Niagara Falls, Ont., pp: 409-411. DOI: 10.1109/AINAW.2007.50

14. Perkins, H.E. and E.M. Royer, 1999. Ad-hoc ondemand distance vector routing. Proceeding of the 2nd IEEE Workshop on Mobile Computing Systems and Applications, Feb. 25-26, IEEE Xplore Press, New Orleans, LA, USA., pp: 90-100. DOI: 10.1109/MCSA.1999.749281

15. Perkins, A.E. and P. Bhagwat, 1994. Highly dynamic destination-sequenced distance vector routing (dsdv) for mobile computers. Comput. Commun. Rev., 24: 234-244. http://portal.acm.org/citation.cfm?id=190336

16. Jacquet, P., P. Mahlethaler and A. Qayyum, 2003. Optimized link state routing protocol, IETF MANET, Internet Draft. http://www.ietf.org/rfc/rfc3626.txt

17. Johnson, D.B. and D.A. Maltz, 1996. Dynamic Source Routing in Ad Hoc Wireless Networks. In: Mobile Computing, Imielinski, T. and H. Korth (Eds.). Kluwer Publishing Company, USA., Ch. 5, ISBN: 0792396979, 9780792396970, pp: 153-181.

18. Douglas, S.J., De Couto, D.A. Yo, J. Bicket and R. Morris, 2005. A high-throughput path metric for multi-hop wireless routing. Wireless Networks, 11: 419-434.

http://portal.acm.org/citation.cfm?id=1150541

19. Draves, R., J. Padhye and B. Zill, 2004. Routing in multi-radio, multi-hop wireless networks. Proceedings of the 10th Annual International Conference on Mobile Computing and Networking, Sept. 26-Oct. 01, Philadekphia, USA., pp: 114-128.

http://portal.acm.org/citation.cfm?id=1023732

20. Yang, Y., J. Wang and R. Kravets, 2006. Interference-aware load balancing for Multihop Wireless Networks, Technical Report UIUCDCSR-2005-2526, Department of Computer Science, University of Illinois at Urban-Champaign. http://whitepapers.techrepublic.com.com/abstract.a spx?docid $=375148$

21. Manoj, B.S. and R.R. Rao, 2007. Wireless Mesh Networks: Issues and Solutions. In: Wireless Mesh Networking: Architectures, Protocols and Standards, Yan Zhang, Jijun Luo and Honglin $\mathrm{Hu}$ (Eds.). Auerbach Publications, Ch. 1, New York, USA., ISBN: 0849373999, pp: 3-48. 
22. Wang, S. and Z. Qiu, 2006. A link type aware routing metric for wireless mesh networks. Proceeding of the 8th International Conference on Signal Processing, Vol. 4, Nov. 16-20, IEEE Xplore Press, Beijing. DOI: 10.1109/ICOSP.2006.346103

23. Rahman, M.A., M.S. Azad and F. Anwar, 2008. The efficient use of lifetime to enhance the performance of AODV. Proceeding of the 4th International Conference on Information Technology and Multimedia at UNITEN, Nov. 1719, Malaysia, pp. 287-291.

24. Jetcheva, J.G. and D.V. Johson, 2004. A Performance comparison of on-demand multicast routing protocols for ad hoc networks. Report No. A556384.

http://www.stormingmedia.us/55/5563/A556384.html
25. Jorg, D.O., 2003. Performance comparison of MANET Routing protocols in different network sizes, computer science project. University of Berne, Switzerland. http://www.iam.unibe.ch/ rvs/research/publication s/projekt_david_joerg.pdf

26. Nguyen, U.T. and X. Xiong, 2005. Rate-adaptive multicast in mobile ad-hoc networks. Proceeding of the IEEE International Conference on Ad hoc and Mobile Computing, Networking and Communications, Aug. 22-24, IEEE Xplore Press, Montreal, Canada, pp: 352-360. DOI: 10.1109/WIMOB.2005.1512924 